\title{
Physiologic anchorage Speewire system
}

\section{Tian $\operatorname{Min} \mathbf{X u}^{1}$ iD}

Received: 26 March 2018 / Accepted: 13 June 2018 / Published online: 6 July 2018

(c) The Author(s) 2018

\begin{abstract}
Physiologic anchorage Speewire system (PASS) technique is based on physiologic anchorage control theory. It is based on craniofacial growth, dentoalveolar compensation, physiologic curve of Spee, principle of differential moments, and the advantage of light force low friction. PASS appliance consists of XBT buccal tube and MLF bracket.
\end{abstract}

Keywords Physiologic anchorage loss · Curve of Spee · Anchorage reserve · Differential moments $\cdot$ Light force $\cdot$ Low friction

\section{Indications}

Malocclusion

\section{Materials/instruments}

XBT buccal tube

MLF bracket

\section{Mechanism and procedure}

Orthodontists used to attribute molar mesial displacement (anchorage loss) during orthodontic extraction treatment to reaction force of anterior teeth retraction. Our RCT study showed the amount of anchorage loss was not only affected by orthodontic retraction technique but also by growth rhythm and gender. Craniofacial longitudinal growth study also shows upper molar tips forward during

Tian Min Xu

tmxuortho@163.com

1 Orthodontic Department, Peking University School of Stomatology, 22 Zhongguancun South Av. Haidian District, Beijing 100081, China 
growth as the compensation of mandible outgrows the maxilla. So we divided the anchorage loss during orthodontic treatment into two parts: one by orthodontic appliance-the mechanical anchorage loss; the other by physiologic force such as growth, occlusal force, periodontal ligament force, etc.-physiologic anchorage loss. The concept of physiologic anchorage control is if we can prevent upper molar forward tipping growth from the first NiTi archwire in aligning stage, do not level or restore upper posterior curve of Spee (it actually indicates upper buccal teeth gradually tipped back from premolar to the last molar) during following treatment, it will not only provide an strong anchorage similar to Tweed anchorage preparation mechanism in retraction stage, but also save more room to retract anterior teeth by aligning posterior teeth on a curved archwire rather than on a straight archwire. So we call this physiologic anchorage Speewire system (PASS).

In PASS, physiologic features of dentition are followed as much as possible and these features are used during orthodontic treatment. In this technique, the physiologic or compensated curve of Spee is kept in the posterior segment for function while straightening anterior teeth for esthetics.

The basic principle of physiologic anchorage control (PAC) technique is moving the teeth to the position in harmony with oral physiologic characteristics with PASS appliance.

The appliance is designed to reduce unfavorable force system in appliance and to take advantage of all physiologic forces in the mouth.



Fig. 1 Physiologic anchorage loss caused by mandible growth and U7 eruption 
Harmonious position can be considered as:

- Roots must be within alveolus.

- Upper arch should have the normal curve of Spee, especially in adolescents.

- The nature compensation of posterior teeth to abnormal skeletal relationship is considered.

- The functional occlusal plane affected by posterior teeth orientation should be determined by oral-gnathic function of individuals.

In PAC system, the largest or dominant moment is on the anchor molar as its direction can be determined according to Burstone's six geometric classes between two neighboring teeth.

The PASS considers following forces and movements in the treatment mechanics:

- Forces from muscles of lip, buccal area, and tongue

- Tooth eruption force

- Bite force

- Periodontal ligament force

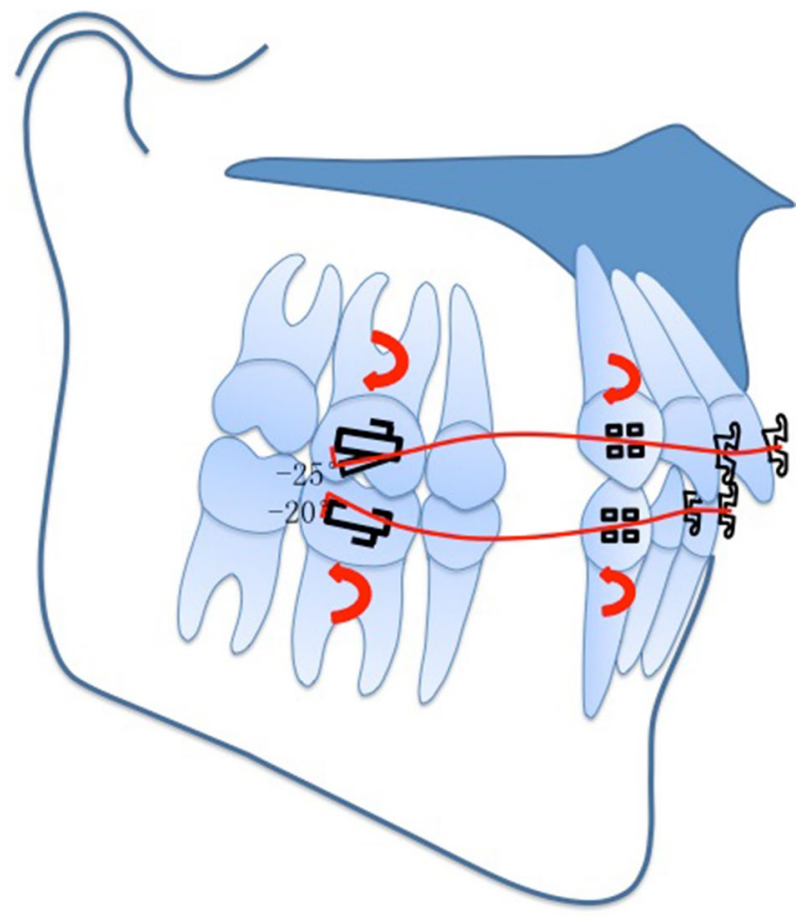

Fig. 2 Designed dominant moments on anchor molars may not only prevent PAL, but also facilitate canine backward "drifting" on first thin NiTi archwire 


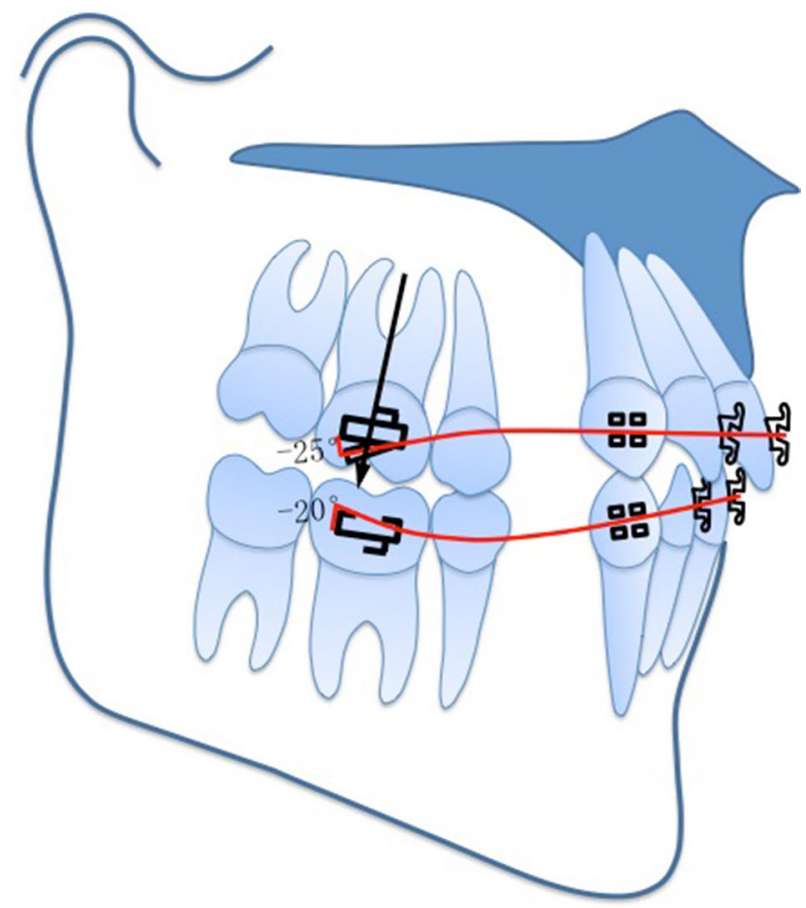

Fig. 3 The forward-tipping compensation of upper first molar is resisted by first NiTi wire in aligning stage, although it may still erupt along its long axis to compensate jaw differential growth rotation, which is supposed to be good for upper molar apex moving downward and backward to increase arch length

In PASS technique (Figs. 1, 2, 3 and 4):

- Taking first premolar extraction case as an example: according to physiologic anchorage loss theory, upper posterior teeth will tip forward with mandible forward growth (Fig. 1).

- Molar XBT tube and anterior MLF brackets are bonded within 2 weeks after extraction (Fig. 2).

- Initial thin NiTi wires are engaged in $-25^{\circ}$ tip back tube on upper first molar and $-20^{\circ}$ tip back "virtual tube" on lower first molar (Fig. 2). According to Burstone's six geometric classes, the larger moment will be on anchor molar because of the larger wire-slot angle. Canines in most cases will get the same direction but smaller moment if they are closed to normal angulation, so they will be tipped backward to relieve anterior crowding without laceback or tieback. The backward tipping moment on molar can only prevent molar forward tipping but cannot stop molar eruption with growth, so it changes upper molar growth pattern from downward and forward to downward and backward along with its long axis (Fig. 3), which may increase dental arch length.

- When malocclusion teeth in the front are corrected to near normal or wire-slot angulation on all other teeth is less than $7^{\circ}$ on upper arch and $4^{\circ}$ on lower arch, then we bond second premolars and second molars, and $-7^{\circ}$ rectangular tube on 


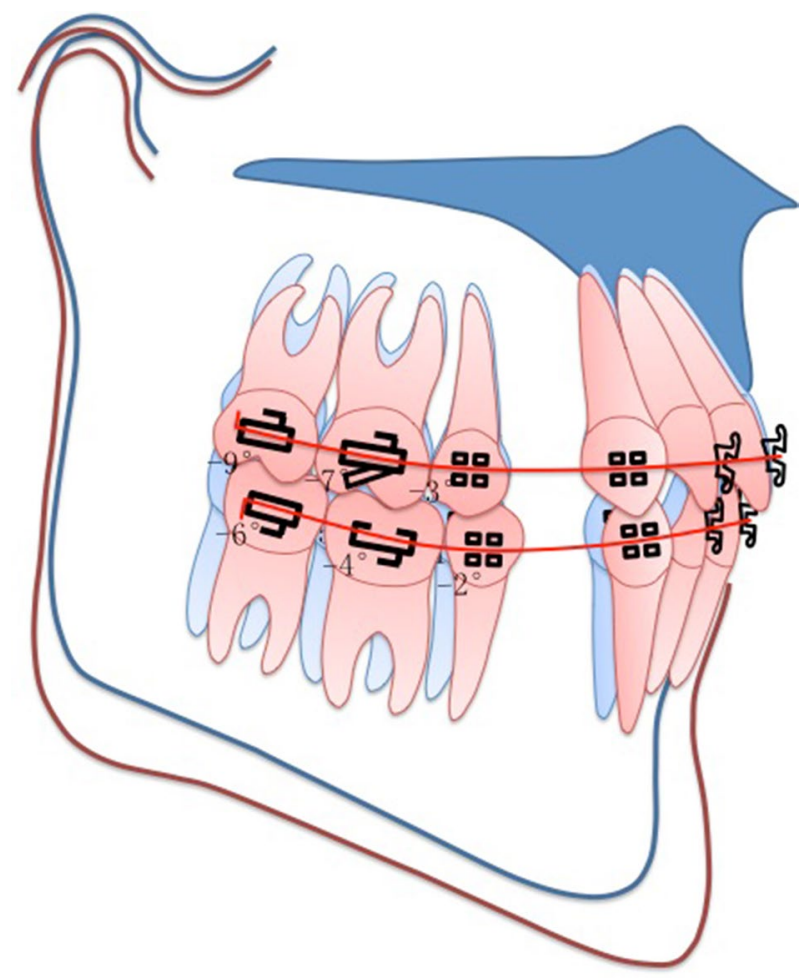

Fig. 4 All posterior brackets or tubes are in tip back angle to resist the compensation of upper arch to mandible growth during whole treatment period. The upper archwires with normal or compensated curve of Spee are applied to avoid leveling upper posterior curve

the upper first molar and $-4^{\circ}$ rectangular tube on the lower first molar are used (Fig. 4). In this way, the larger moment or dominant moment of the system is still on posterior teeth or anchor teeth. From this stage, all upper arch wires need normal or compensated curve of Spee to avoid leveling upper posterior curve of Spee, and lower archwire usually needs reverse curve of Spee to open the bite and enhance posterior anchorage until all extraction space is closed.

\section{Pitfalls and complications}

When straight NiTi wire is used to align irregular teeth, prediction of the force on each tooth becomes difficult.

Funding This article received funding from International Science and Technology Cooperation Program of China under Grant no. 2014DFA318.

Open Access This article is distributed under the terms of the Creative Commons Attribution 4.0 International License (http://creativecommons.org/licenses/by/4.0/), which permits unrestricted use, distribution, 
and reproduction in any medium, provided you give appropriate credit to the original author(s) and the source, provide a link to the Creative Commons license, and indicate if changes were made.

\section{Further reading}

1. Xu TM (ed) Physiologic anchorage control, https://doi.org/10.1007/978-3-319-48333-7_8 Physiologic Anchorage Control System

2. Bjork A, Skieller V (1972) Facial development and tooth eruption. An implant study at the age of puberty. Am J Orthodontics 62(4):339-383

3. Burstone CJ, Koenig HA (1974) Force systems from an ideal arch. Am J Orthod 65(3):270-289

4. McKinney JR, Harris EF (2001) Influence of patient age and sex on orthodontic treatment: evaluations of Begg lightwire, standard edgewise, and straight wire techniques. Am J Orthod Dentofac Orthop 120(5):530-541

5. Martinelli FL, Ruellas AC, Lima EM et al (2010) Natural changes of the maxillary first molars in adolescents with skeletal Class II malocclusion. Am J Orthod Dentofac Orthop 137(6):775-781

6. Xu TM, Zhang X, Oh HS et al (2010) Randomized clinical trial comparing control of maxillary anchorage with 2 retraction techniques. Am J Orthod Dentofac Orthop 138(5):544.e1-9

7. Su H, Han B, Li S, Na B, Ma W, Xu TM (2014) Factors predisposing to maxillary anchorage loss: a retrospective study of 1403 cases. PLoS One 9(10):e109561 